\section{The prevalence and correlates of childhood trauma in patients with early psychosis}

Australian \& New Zealand Journal of Psychiatry $1-9$ DOI: $10.1177 / 0004867415575379$

(C) The Royal Australian and New Zealand College of Psychiatrists 2015 Reprints and permissions: sagepub.co.uk/journalsPermissions.nav anp.sagepub.com (\$SAGE

\author{
Michael Duhig'1,2,3, Sue Patterson 4,5, Melissa Connell2, \\ Sharon Foley ${ }^{6}$, Carina Capra ${ }^{6}$, Frances Dark ${ }^{6}$, Anne Gordon 4 , \\ Saveena Singh', Leanne Hides ${ }^{3,8}$, John J McGrath' ${ }^{1,9}$ and \\ James Scott ${ }^{1,2,4,10}$
}

\title{
Abstract
}

Objective: To describe the prevalence and demographic, clinical and functional correlates of childhood trauma in patients attending early psychosis clinics.

Method: Participants were recruited from outpatients attending four early psychosis services. Exposure to childhood trauma was assessed using the Childhood Trauma Questionnaire (CTQ). Psychopathology was measured using the Positive and Negative Syndrome Scale and the Depression, Anxiety and Stress Scale. Social and vocational functioning and substance use were also assessed.

Results: Over three-quarters of the 100 patients reported exposure to any childhood trauma. Emotional, physical and sexual abuse were reported by $54 \%, 23 \%$ and $28 \%$ of patients, respectively, while $49 \%$ and $42 \%$ of patients reported emotional and physical neglect, respectively. Female participants were significantly more likely to be exposed to emotional and sexual abuse. Exposure to childhood trauma was correlated with positive psychotic symptoms and higher levels of depressive, anxiety and stress symptoms; however, it had no impact on social or vocational functioning or recent substance use.

Conclusion: Exposure to childhood trauma was common in patients with early psychosis, and associated with increased symptomatology. Existing recommendations that standard clinical assessment of patients with early psychosis should include inquiry into exposure to childhood trauma are supported.

Keywords

prevalence, psychotic disorders, psychosis, child abuse, substance abuse

\footnotetext{
'Queensland Centre for Mental Health Research, The Park Centre for Mental Health, Wacol, QLD, Australia

${ }^{2}$ The University of Queensland Centre for Clinical Research, Herston, QLD, Australia

${ }^{3}$ School of Psychology and Counselling, Queensland University of Technology, Kelvin Grove, QLD, Australia

${ }^{4}$ Metro North Mental Health, Royal Brisbane and Women's Hospital, Herston, QLD, Australia

${ }^{5}$ School of Applied Psychology, Griffith University, Mt Gravatt, QLD, Australia

6Metro South Mental Health, Princess Alexandra Hospital, Wooloongabba, QLD, Australia

${ }^{7}$ Early Psychosis Service, Gold Coast Health Service District, Burleigh Heads, Gold Coast, QLD, Australia

8Institute of Health and Biomedical Innovation, School of Psychology and Counselling, Queensland University of Technology, Kelvin Grove, QLD,

Australia

${ }^{9}$ Queensland Brain Institute, University of Queensland, St Lucia, QLD, Australia

${ }^{10}$ Discipline of Psychiatry, University of Queensland, St Lucia, QLD, Australia

Corresponding author:

James Scott, Level 3 UQCCR, RBWH, Herston, QLD 4029, Australia.

Email: james.scott@health.qld.gov.au
} 


\section{Introduction}

Childhood trauma (CT) can have persistent adverse effects on an individual's physical and mental health, social development and wellbeing (Norman et al., 2012). Differing descriptions of CT exist throughout the contemporary literature. While many studies define $\mathrm{CT}$ as all forms of physical and/or emotional ill-treatment, sexual abuse, neglect or negligent treatment (Krug et al., 2002; Norman et al., 2012), others have used broader definitions which include bullying and victimisation, parental loss and separation (Varese et al., 2012). For the purposes of this study, CT is defined as physical, sexual and emotional abuse and physical or emotional neglect (Daruy-Filho et al., 2011).

Historically, studies examining the relationship between CT and psychosis have reported inconsistent findings although recently, the association has become widely accepted. For example, a prospective case control study of 1,612 Victorian children identified as being exposed to childhood sexual abuse (CSA) reported increased rates of treatment required for mental illness but no increased risk of schizophrenia (Spataro et al., 2004). However, an extension of this study employing a larger sample $(\mathrm{N}=2,759)$ found those exposed to CSA were 2.1 and 2.6 times more likely to be diagnosed with psychosis and schizophrenia, respectively (Cutajar et al., 2010). The association between $\mathrm{CT}$ and psychotic disorders is now well accepted (Bendall et al., 2008; Matheson et al., 2013; Morgan and Fisher, 2007; Shevlin et al., 2007; Varese et al., 2012).

In a literature review, the prevalence of CT in those with psychosis was reported to range widely from $59 \%$ to $100 \%$ (Read et al., 2005). Similarly, Australian prevalence rates of trauma also vary with physical abuse in the range of $5-10 \%$, neglect in the range of $2-12 \%$ and emotional maltreatment in the range of $6-17 \%$ (Australian Insitiute of Family Studies, 2013). Sexual abuse has been reported to affect $4-16 \%$ of boys and $12-34 \%$ of girls (Dunne et al., 2003). The wide variation in the prevalence of CT is due to the definition of childhood trauma utilised, methods of reporting (e.g. self-report vs. use of child protection agency records), sex and age of the individuals. The use of validated standardised measures of CT is one method of overcoming problems arising from definitional variation.

In spite of the many studies examining the association between CT and psychotic disorders, few have examined CT in patients with early psychosis (EP). Ucok and Bikmaz (2007) evaluated the impact of CT among 57 inpatients with first-episode schizophrenia. Those exposed to childhood sexual abuse reported more severe positive psychotic symptoms and were more likely to have made a recent suicide attempt while those exposed to childhood emotional abuse reported more hallucinations and delusions. Similarly, Bendall et al. (2013b) reported that EP patients exposed to childhood sexual abuse had more severe hallucinations and delusions, and Wang and colleagues (2013) found CT was significantly correlated with hallucinations $(r=0.174$, $P=0.049)$ in EP patients but not patients with chronic psychosis. Finally, a study by Ramsay et al. (2011) found correlations between childhood emotional abuse and neglect and positive and negative psychotic symptoms, respectively, in disadvantaged, African-American EP inpatients.

The impact of CT on other symptom domains of patients with EP is not well established. While depression, anxiety and stress symptoms have been shown to be higher in patients with persistent psychotic disorders exposed to CT (Lysaker and Salyers, 2007; Schenkel et al., 2005), no studies have examined this association in patients with EP. By contrast, increased rates of lifetime substance abuse have been reported in EP populations with a history of CT (Schafer and Fisher, 2011). A study of 61 EP inpatients with concurrent substance use found $85 \%$ had a history of CT (Fraser et al., 2012) while Ramsay et al. (2011) reported CT was associated with lifetime cannabis use and a younger age of onset of alcohol use. Although social and vocational functioning has been identified as a key treatment outcome for clinicians (Figueira and Brissos, 2011) and patients with psychosis (Stain et al., 2012), very few studies have examined the impact of CT on functioning in EP patients. Poorer premorbid functioning was associated with a history of sexual and/or physical abuse in first-episode mania (Conus et al., 2010) and a history of any CT in 102 EP patients (Stain et al., 2013). In summary, there are few studies examining the impact of CT on the clinical presentation and functioning of patients with EP. Those available suggest patients with a history of CT are more symptomatic and have poorer levels of premorbid functioning.

This study aimed to establish the prevalence of CT in patients attending EP outpatient services in an Australian city. A further aim was to examine the demographic, clinical and functional correlates of childhood trauma in EP patients. It was hypothesised that exposure to CT would be associated with increased positive and negative psychotic symptomatology and higher levels of anxiety, depression and stress. Furthermore, we hypothesised that those exposed to CT would report greater levels of substance use and have poorer levels of social and vocational functioning.

\section{Method \\ Participants}

Participants were recruited from four specialist EP outpatient clinics in South-East Queensland, Australia. Patients attending these services were in the age range 15-25 years, diagnosed with a psychotic illness and had not received greater than 2 years of mental health treatment for psychosis. Participants were informed of the aims of the study by their treating clinician and those willing to participate provided written consent. 


\section{Instruments}

Demographic and clinical information. Demographic data including age, sex, relationship status, country of birth, language spoken at home, ethnicity, dependents and employment status were collected using a standardised questionnaire completed by participants. Clinical data collected were primary and secondary diagnoses made by a consultant psychiatrist using the 10th Edition of the International Classification of Diseases (ICD-10) (World Health Organization, 1992) and estimated duration of untreated and treated psychosis which was verified by treating clinicians and clinical records. Duration of untreated psychosis (DUP) was defined as the time between onset of psychotic symptoms as reported by participants or carers and first contact with psychiatric services.

Psychotic symptoms. The Positive and Negative Syndrome Scale (PANSS) is an interviewer-rated scale measuring psychotic and general symptoms in patients with psychosis. Prior to initiating the study, clinicians were trained in the PANSS by an investigator experienced in using the instrument. Clinicians were required to obtain an inter-rater reliability score of $\alpha=0.80$ or greater using the PANSS on a videotaped clinical interview before being permitted to collect data for the study.

Childhood trauma. The 28-item Childhood Trauma Questionnaire (CTQ) was used to assess levels of exposure to childhood abuse and neglect (Bernstein, 1998; Scher et al., 2001). It was chosen as it examines emotional, physical and sexual abuse, as well as emotional and physical neglect and yields an overall childhood trauma score. Each item is scored on a 5 point Likert scale. An example of an item is 'Someone tried to make me do sexual things or watch sexual things' to which responses range from 'never true' (1) to 'very often true' (5), producing scores in the range of 5 to 25 for each trauma sub-scale and 25 to 125 for the overall trauma score. Trauma prevalence was estimated using thresholds described in the CTQ manual (Bernstein, 1998). Sub-scale classifications of none to minimal were categorised as trauma absent while sub-scale classifications of minimal to moderate, moderate to severe and severe to extreme were categorised as trauma present. For correlation with clinical measures data remained in continuous form as used in previous research (Ucok and Bikmaz, 2007).

Depression, anxiety and stress. The depression, anxiety and stress scale (DASS21) is a 21-item self-report measure examining symptoms in these three domains over the previous seven days (Lovibond and Lovibond, 1995). Total scores for each subscale were used for correlation analysis.

Social and vocational functioning. The Activity and Participation Questionnaire (APQ6) is a retrospective, self-report instrument assessing vocational activity and social participation in the previous 7 days (Stewart et al., 2010). Measures of vocational activity include hours spent in current employment, studying, job seeking and volunteer work. Social participation measures include hours spent engaged in social activities (e.g. visiting a friend or relative or participation in a sporting or recreational group). For statistical analysis, patients were categorised dichotomously as vocationally active if they were employed or studying for a minimum of 1 hour in the previous 7 days, or not in education, employment or training (NEET). Social functioning within the previous 7 days was categorised into three groups: absent; low; and high. Patients who did not engage in any social activity were classed as absent, those participating in social activities for between 1 and 6 hours were categorised as low functioning and patients who engaged in 7 or more hours were classed as high functioning.

Current and lifetime substance use questionnaire. Participants' substance use over the previous 28 days was rated by clinicians using a standardised questionnaire. Levels of use were categorised into four groups: no use (no substance use within the last month); infrequent use (used once to twice in the previous month); regular use (used one to twice per week for the previous month); and frequent use (used three or more times per week for the previous month). For statistical analysis, substances were grouped into four forms: alcohol; tobacco; cannabis; and other illicit drugs. Illicit drugs included amphetamines, ecstasy, opiates, benzodiazepines, volatile substances (e.g. inhalants, glue, petrol) and other drugs (e.g. ecstasy, cocaine, LSD).

\section{Procedure}

Following ethics approval, patients identified by their treating clinician as being able to provide informed consent were invited to participate in the study. We have previously reported an analysis of this recruitment strategy revealing clinicians were more likely to approach patients who were less symptomatic and had better insight (Patterson et al., 2014). Patients who agreed to participate were more likely to be actively engaged in treatment (Patterson et al., 2014). Consent of both participants and guardians was obtained for those aged under 18 years. The PANSS, APQ6 and substance use questionnaire were administered by PANSStrained clinicians. Following this, participants completed self-report questionnaires (CTQ and DASS21). Participants received a gift card for participating. No information was available on those patients who refused to participate or who were not approached.

\section{Statistical analysis}

As CTQ scores were not normally distributed, associations between CTQ scores, positive and negative symptom 
scores and DASS21 responses were examined using Spearman's correlations. Positive and negative symptom scores were transformed from interval to ratio data by subtracting the theoretical minimum ( 7 points) from each score (Obermeier et al., 2011). The association between CTQ scores and vocational functioning was examined using Mann-Whitney U-tests with a median split used to define groups. Analysis of the relationship between CTQ scores and social functioning was completed using Kruskal-Wallis $\mathrm{H}$-tests also using a median split used to analyse groups. Univariate ANOVA calculations with bootstrapping were used to examine the association between CTQ scores and substance use in the past month. Tests of significance were two-tailed, Holm-Bonferroni corrections were implemented in post-hoc analysis where applicable and the software used to perform analyses was IBM SPSS for Windows version 22.0 (IBM Corporation, 2013).

\section{Results}

One hundred and six patients consented to participate; however, only 100 completed all measures and were included in final analysis. This sample consisted of 62 men and 38 women with a mean age of 21.4 years $(S D=2.28$, range $=17-26$ years) and a median DUP of 4 weeks $(S D=44.4$, range $=1-266$ weeks $)$. Eighty-seven percent were single, 76\% were born in Australia and 93\% had no dependents. Fifty-six percent were unemployed, 12\% were employed part-time while 9\% were employed full-time. A further $18 \%$ were engaged in educational activities (university or technical college). Schizophrenia was the most common diagnosis $(32 \%)$ followed by substance-induced psychotic disorder (18\%) and psychotic disorder not otherwise specified (17\%). A complete list of diagnoses for the total sample and by sex is reported in Table 1. Men were more frequently diagnosed with schizophrenia while women were more likely to receive a diagnosis of psychotic disorder not otherwise specified.
More than three-quarters $(\mathrm{N}=82)$ of patients receiving treatment from an EP clinic reported exposure to any form of abuse or neglect, with 64 patients $(64 \%)$ exposed to at least one form of abuse while 62 patients (62\%) were exposed to one or more forms of neglect. Sex differences were observed with women significantly more likely to report exposure to emotional and sexual abuse (Table 2) and this abuse was more severe in women (Table 3 ).

The total CTQ score was significantly positively correlated with positive psychotic symptoms only while physical neglect was significantly correlated with both positive and negative psychotic symptoms. After controlling for multiple comparisons, correlations between total CTQ score and positive symptoms and physical neglect and negative symptoms did not reach statistical significance. This was most likely due to the restricted sample size and limited power for multiple comparisons. There was a trend towards significance of emotional neglect also being correlated with both psychotic symptom domains. Exposure to other types of CT was not significantly correlated with positive or negative psychotic symptoms (Table 4). When stratified by sex, physical neglect was correlated with both positive and negative psychotic symptoms in men and women (Supplementary Table 1).

Total CTQ scores were significantly positively correlated with all forms of emotional distress. Exposure to emotional or physical abuse or physical neglect were correlated with increased depression, anxiety and stress symptoms while sexual abuse was correlated with increased symptoms of depression only (Table 5). The correlations between trauma exposure and emotional distress were very similar when the sample was stratified (Supplementary Table 2).

Forty-five percent of patients were vocationally active in the previous 7 days. Exposure to CT was not significantly associated with vocational activity in patients with EP overall (Supplementary Table 3) or when stratified by sex (Supplementary Table 4). Six percent of patients did not engage in any social activity within the previous 7 days,

Table I. Frequency of Axis I diagnoses overall and by sex.

\begin{tabular}{|lccc|}
\hline Diagnosis & Overall $(\%)$ & Men $(\%)$ & Women (\%) \\
\hline Schizophrenia & $32(32)$ & $23(37.1)$ & $9(23.7)$ \\
\hline Psychosis not otherwise specified & $18(18)$ & $8(12.9)$ & $10(26.3)$ \\
\hline Substance-induced psychosis & $17(17)$ & $11(17.7)$ & $6(15.8)$ \\
\hline Bipolar affective disorder & $15(15)$ & $8(12.9)$ & $7(18.4)$ \\
\hline Schizophreniform psychosis & $10(10)$ & $7(11.3)$ & $3(7.9)$ \\
\hline Schizo-affective disorder & $6(6)$ & $5(8.1)$ & $1(2.6)$ \\
\hline Major depressive disorder with psychosis & $2(2)$ & 0 & $2(5.3)$ \\
\hline Total & $100(100)$ & $62(100)$ & $38(100)$
\end{tabular}


Table 2. Rates of trauma in patients with early psychosis.

\begin{tabular}{|llllll|}
\hline CTQ variable & Total $(\mathrm{N}=100)$ & Men $(\mathrm{N}=62)$ & Women $(\mathrm{N}=38)$ & $\chi 2$ & P value \\
\hline Emotional abuse & $54(54 \%)$ & $27(43.5 \%)$ & $27(71.1 \%)$ & $\mathbf{7 . 1 8 * *}$ & 0.007 \\
\hline Physical abuse & $23(23 \%)$ & $13(21 \%)$ & $10(26.3 \%)$ & 0.38 & 0.537 \\
\hline Sexual abuse & $28(28 \%)$ & $12(19.4 \%)$ & $16(42.1 \%)$ & $6.05 *$ & 0.014 \\
\hline Emotional neglect & $49(49 \%)$ & $27(43.5 \%)$ & $22(57.9 \%)$ & 1.94 & 0.164 \\
\hline Physical neglect & $42(42 \%)$ & $24(38.7 \%)$ & $18(47.4 \%)$ & 0.73 & 0.394 \\
\hline
\end{tabular}

CTQ: Childhood trauma questionnaire.

$* P<0.05$.

$* * P<0.01$

Table 3. Distribution of trauma severity overall and by sex.

\begin{tabular}{|c|c|c|c|c|c|}
\hline & Overall $(\mathrm{N}=100)$ & Men $(\mathrm{N}=62)$ & Women $(\mathrm{N}=38)$ & Exp B $(95 \% \mathrm{Cl})$ & $P$ value \\
\hline \multicolumn{6}{|l|}{ Emotional abuse } \\
\hline None to minimal & 46 (46\%) & 35 (56.5\%) & II (28.9\%) & Reference category & \\
\hline Minimal to moderate & $30(30 \%)$ & I 8 (29\%) & $12(3 \mid .6 \%)$ & $0.47(0.17-1.28)$ & 0.139 \\
\hline Moderate to severe & II (II\%) & $5(8 \%)$ & $6(15.8 \%)$ & $0.26(0.67-1.03)$ & 0.055 \\
\hline Severe to extreme & $13(13 \%)$ & $4(6.5 \%)$ & $9(23.7 \%)$ & $0.14(0.04-0.54)$ & $0.005 * *$ \\
\hline \multicolumn{6}{|l|}{ Physical abuse } \\
\hline None to minimal & 77 (77\%) & 49 (79\%) & $28(73.7 \%)$ & Reference category & \\
\hline Minimal to moderate & $6(6 \%)$ & $4(6.5 \%)$ & $2(5.3 \%)$ & $1.14(0.20-6.64)$ & 0.882 \\
\hline Moderate to severe & 7 (7\%) & $6(9.7 \%)$ & I (2.6\%) & $3.43(0.40-29.95)$ & 0.265 \\
\hline Severe to extreme & $10(10 \%)$ & $3(4.8 \%)$ & $7(18.4 \%)$ & $0.25(0.59-1.02)$ & 0.054 \\
\hline \multicolumn{6}{|l|}{ Sexual abuse } \\
\hline None to minimal & 72 (72\%) & $50(80.6 \%)$ & 22 (57.9\%) & Reference category & \\
\hline Minimal to moderate & $8(8 \%)$ & $6(9.7 \%)$ & $2(5.3 \%)$ & $1.32(0.25-7.06)$ & 0.746 \\
\hline Moderate to severe & $9(9 \%)$ & $3(4.8 \%)$ & $6(15.8 \%)$ & $0.22(0.05-0.96)$ & $0.044^{*}$ \\
\hline Severe to extreme & II (II\%) & $3(4.8 \%)$ & $8(21.1 \%)$ & $0.17(0.40-0.68)$ & $0.013 *$ \\
\hline \multicolumn{6}{|l|}{ Emotional neglect } \\
\hline None to minimal & $5 I(51 \%)$ & $35(56.5 \%)$ & $16(42.1 \%)$ & Reference category & \\
\hline Minimal to moderate & 33 (33\%) & I 8 (29\%) & $15(39.5)$ & $0.55(0.22-1.36)$ & 0.194 \\
\hline Moderate to severe & $10(10 \%)$ & 7 (1 I.3\%) & $3(7.9 \%)$ & $1.07(0.24-4.67)$ & 0.932 \\
\hline Severe to extreme & $6(6 \%)$ & $2(3.2 \%)$ & $4(10.5 \%)$ & $0.29(0.38-1.38)$ & 0.108 \\
\hline \multicolumn{6}{|l|}{ Physical neglect } \\
\hline None to minimal & $58(58 \%)$ & $38(62.3 \%)$ & $20(52.6 \%)$ & Reference category & \\
\hline Minimal to moderate & $19(19 \%)$ & $12(19.4 \%)$ & 7 (I8.4\%) & $0.90(0.31-2.65)$ & 0.852 \\
\hline Moderate to severe & $13(13 \%)$ & $8(12.9 \%)$ & $5(13.2 \%)$ & $0.84(0.24-2.92)$ & 0.786 \\
\hline Severe to extreme & $10(10 \%)$ & $4(6.5 \%)$ & $6(15.8 \%)$ & $0.35(0.09-1.39)$ & 0.136 \\
\hline
\end{tabular}

whereas 39\% engaged in low levels and 53\% engaged in high levels of social functioning. There was no statistically significant difference in mean scores for any trauma or total CTQ score for each level of social functioning overall (Supplementary Table 5) or when stratified by sex (Supplementary Table 6).
In the previous month, both alcohol and tobacco were used by $67 \%$ of patients while $27 \%$ reported using cannabis and $17 \%$ reported illicit drug use. No associations between exposure to CT and the level of tobacco, alcohol, cannabis or other illicit drugs used in the past month were found (Supplementary Tables 7 and 8). When stratified by sex, no 
Table 4. Correlations of childhood trauma and positive and negative psychotic symptoms.

\begin{tabular}{|c|c|c|c|c|}
\hline \multirow[b]{3}{*}{ CTQ variable } & \multicolumn{4}{|l|}{ PANSS } \\
\hline & \multicolumn{2}{|c|}{ Positive symptoms } & \multicolumn{2}{|c|}{ Negative symptoms } \\
\hline & rs & $P$ value & rs & $P$ value \\
\hline Emotional abuse & 0.17 & 0.083 & -0.02 & 0.843 \\
\hline Physical abuse & 0.08 & 0.406 & -0.02 & 0.872 \\
\hline Sexual abuse & 0.02 & 0.852 & -0.14 & 0.159 \\
\hline Emotional neglect & 0.19 & 0.057 & 0.18 & 0.070 \\
\hline Physical neglect & $0.32 * * \dagger$ & $<0.01$ & $0.25 *$ & 0.013 \\
\hline Total CTQ score & $0.23 *$ & 0.022 & 0.06 & 0.550 \\
\hline
\end{tabular}

CTQ: Childhood trauma questionnaire, PANSS: Positive and negative syndrome scale. $* P<0.05$.

$* * P<0.01$

†Significant after controlling for multiple comparisons.

Table 5. Correlation of childhood trauma and depression, anxiety and stress symptoms.

\begin{tabular}{|c|c|c|c|c|c|c|}
\hline \multirow[b]{3}{*}{ CTQ variable } & \multicolumn{6}{|l|}{ DASS } \\
\hline & \multicolumn{2}{|l|}{ Depression } & \multicolumn{2}{|l|}{ Anxiety } & \multicolumn{2}{|l|}{ Stress } \\
\hline & rs & $P$ value & rs & $P$ value & rs & $P$ value \\
\hline Emotional abuse & $0.37 * * * \dagger$ & $<0.001$ & $0.33 * * \dagger$ & 0.001 & $0.32 * * \dagger$ & 0.001 \\
\hline Physical abuse & $0.34 * * \dagger$ & 0.001 & $0.28 * *+$ & 0.005 & $0.32 * * \dagger$ & 0.001 \\
\hline Sexual abuse & $0.20 *$ & 0.042 & 0.14 & 0.153 & 0.14 & 0.154 \\
\hline Emotional neglect & 0.15 & 0.125 & 0.17 & 0.091 & 0.16 & 0.125 \\
\hline Physical neglect & $0.22 *$ & 0.028 & $0.32 * * \dagger$ & 0.001 & $0.25 * t$ & 0.011 \\
\hline Total CTQ score & $0.36 * * \dagger$ & $<0.001$ & $0.36 * * * \dagger$ & $<0.001$ & $0.36 * * \dagger$ & 0.001 \\
\hline
\end{tabular}

CTQ: Childhood trauma questionnaire, DASS: Depression, anxiety and stress scale.

$* P<0.05$.

**P $<0.01$.

$* * * p<0.001$.

†Significant after controlling for multiple comparisons.

correlations were observed for either sex regarding alcohol use (Supplementary Table 9), tobacco use (Supplementary Table 10), cannabis use (Supplementary Table 11) and illicit drug use (Supplementary Table 12).

\section{Discussion}

This observational study reports more than three-quarters of patients attending early psychosis services in Queensland, Australia had been exposed to CT, a prevalence comparable to other samples of EP patients (Ramsay et al., 2011; Ucok and Bikmaz, 2007; Wang et al., 2013). Compared to Australian population samples (Australian Insitiute of Family Studies, 2013), prevalence rates of all forms of CT are increased in patients with EP. Contrasting previous EP studies, women were significantly more likely to report childhood sexual and emotional abuse than men. The increased rate of sexual abuse for women is consistent with a meta-analysis reporting Australian women in the general population are three times more likely to be sexual abused compared to males (Stoltenborgh et al., 2011). Patients with early psychosis exposed to CT were more likely to have higher levels of positive psychotic symptoms and depression, anxiety and stress symptoms. After controlling for multiple comparisons, the association between total CTQ and positive symptoms did not reach statistical significance most likely due to a lack of power leading to a type II error. Depression in those with EP has been 
associated with poorer outcomes (Challis et al., 2013), increased risk of suicidal ideation and deliberate self-harm (Upthegrove et al., 2010).

Conversely, EP patients exposed to CT did not have lower levels of social or vocational functioning or increased risk of recent substance use. Although people exposed to $\mathrm{CT}$ in the general population have higher rates of substance use (Afifi et al., 2012), substance use is also elevated among those with EP ( Barnett et al., 2007; Sevy et al., 2001). As such, the lack of association between substance use and CT in this clinical sample is most likely due to the mediating effect of EP.

Emotional abuse was prevalent in this sample with 54\% of all participants and $71.1 \%$ of girls reporting this experience during childhood. These rates are consistent with those previously reported in patients with early psychosis (Bendall et al., 2012; Ucok and Bikmaz, 2007), though much more common than other populations attending health services. For example, Hollingsworth et al. (2012) reported that $37 \%$ of women $(\mathrm{N}=239)$ attending an antenatal service in the same city as the current study experienced childhood emotional abuse as measured by the CTQ. The high rates of emotional abuse in this study are consistent with previous reports suggesting that childhood trauma is a risk factor for psychosis (Varese et al., 2012).

Consistent with previous studies of EP populations, total CTQ scores were correlated with positive psychosis symptom scores (Ucok and Bikmaz, 2007; Wang et al., 2013). This association was driven in particular by neglect and emotional abuse. Relative to other forms of childhood maltreatment, exposure to neglect and emotional abuse during childhood adversely impacts adolescent mental health (Mills et al., 2013) and are associated with personality and mood disorders during adulthood (Carr et al., 2013). Neglect, in particular, has been shown to increase the risk of atypical neurodevelopment (Glaser, 2014; Panzer, 2008). It is therefore not surprising that neglect and emotional abuse were correlated with increased positive symptoms.

Several theories have been proposed to explain the relationship between CT and positive psychotic symptoms. One such theory is that post-traumatic stress disorder (PTSD) and psychosis involve shared mechanisms such as dissociation, attributional style and interpretation of intrusions ( Bendall et al., 2013b; Morrison et al., 2003). Post-traumatic intrusions may be misattributed by individuals to an external source and are subsequently experienced as hallucinations (Bentall, 2003; Bentall and Fernyhough, 2008). Another theory proposes exposure to CT is associated with both an externalising attribution style and a tendancy to perceive the world as hostile (Bentall and Fernyhough, 2008; Johns et al., 2014). Psychological interventions for psychcotic symtoms (Thomas et al., 2014) in those who have experienced CT are infromed by these theoretical frameworks.

Consistent with previous reports, physical neglect was correlated with negative psychosis symptom scores (van
Dam et al., 2014) . Multiple hypotheses have been suggested for this relationship with most postulating the association is a result of an abstinence from stimulating experiences as a child leading to psychosocial deficits (Colvert et al., 2008). These deficits have been associated with increased levels of positive and negative symptoms in those with EP (Addington et al., 2003) and schizophrenia (Gallagher and Jones, 2013).

The most significant limitation of this study was the aforementioned recruitment biases (Patterson et al., 2014). We were unable to obtain data on those who did not participate and therefore cannot make meaningful comparisons between participants and non-participants. This was a reflection of undertaking observational research in realworld clinical services. The other significant limitation was the use of a self-report measure of CT which could not be independently verified. However, the CTQ is a well validated, standardised instrument for measuring CT and previous research has shown good reliability of self-reporting CT in those with psychosis (Read et al., 2005) and EP (Fisher et al., 2011).

In spite of the above limitations, this is the first study to examine and show that exposure to CT is associated with increased symptoms of depression, anxiety and stress in those with early psychosis. It supports previous research showing that CT is common in those with early psychosis and associated with higher levels of positive psychotic symptoms (Bendall et al., 2013b; Ramsay et al., 2011; Ucok and Bikmaz, 2007; Wang et al., 2013). With this in mind, incorporating trauma informed care into EP treatment programs for patients who have experienced CT may improve outcomes (Bendall et al., 2013a). However, clinicians are sometimes reluctant to inquire if patients have experienced CT for fear that it may fracture rapport (Bendall et al., 2013a) and information regarding CT is rarely disclosed voluntarily by patients (Read et al., 2006, 2007). Similar to other patient groups, clinicians' understanding of the childhood experiences of patients with early psychosis can improve therapeutic alliance and inform psychotherapeutic interventions (Nafisi and Stanley, 2007). Therefore, given the high prevalence of $\mathrm{CT}$ and the associated psychological distress that occurs in patients with EP, we support previous recommendations (Bendall et al., 2013a; Scott et al., 2007; Ucok and Bikmaz, 2007) that standard clinical care of patients with EP should include inquiry into exposure of CT in order to inform treatment.

\section{Acknowledgements}

The authors would like to thank the clinicians and patients who kindly donated their time during data collection.

\section{Declaration of interest}

The authors report no conflicts of interest. The authors alone are responsible for the content and writing of the paper. 


\section{Funding}

The study was funded by a research grant awarded by the Royal Brisbane and Women's Hospital Foundation and a philanthropic donation from BAP Parking (www.bap.com.au).

\section{References}

Addington J, van Mastrigt S and Addington D. (2003) Patterns of premorbid functioning in first-episode psychosis: initial presentation. Schizophrenia Research 62: 23-30.

Afifi TO, Henriksen CA, Asmundson GJ, et al. (2012) Childhood maltreatment and substance use disorders among men and women in a nationally representative sample. Canadian Journal of Psychiatry 57: 677-686.

Australian Insitiute of Family Studies. (2013) The Prevalence of Child Abuse and Neglect. Canberra: Australian Government.

Barnett JH, Werners U, Secher SM, et al. (2007) Substance use in a population-based clinic sample of people with first-episode psychosis. British Journal of Psychiatry 190: 515-520.

Bendall S, Alvarez-Jimenez M, Hulbert CA, et al. (2012) Childhood trauma increases the risk of post-traumatic stress disorder in response to first-episode psychosis. Australian and New Zealand Journal of Psychiatry 46: 35-39.

Bendall S, Alvarez-Jimenez M, Nelson B, et al. (2013a) Childhood trauma and psychosis: new perspectives on aetiology and treatment. Early Intervention in Psychiatry 7: 1-4.

Bendall S, Hulbert CA, Alvarez-Jimenez M, et al. (2013b) Testing a model of the relationship between childhood sexual abuse and psychosis in a first-episode psychosis group: the role of hallucinations and delusions, posttraumatic intrusions, and selective attention. Journal of Nervous and Mental Disease 201: 941-947.

Bendall S, Jackson HJ, Hulbert CA, et al. (2008) Childhood trauma and psychotic disorders: a systematic, critical review of the evidence. Schizophrenia Bulletin 34: 568-579.

Bentall RP. (2003) Madness Explained. London: Allen Lane.

Bentall RP and Fernyhough C. (2008) Social predictors of psychotic experiences: specificity and psychological mechanisms. Schizophrenia Bulletin 34: 1012-1020.

Bernstein DP and Fink L. (1998) Childhood Trauma Questionnaire Manual. San Antonio, TX: The Psychological Corporation.

Carr CP, Martins CM, Stingel AM, et al. (2013) The role of early life stress in adult psychiatric disorders: a systematic review according to childhood trauma subtypes. Journal of Nervous and Mental Disease 201: $1007-1020$

Challis S, Nielssen O, Harris A, et al. (2013) Systematic meta-analysis of the risk factors for deliberate self-harm before and after treatment for first-episode psychosis. Acta Psychiatrica Scandinavica 127: 442-454.

Colvert E, Rutter M, Kreppner J, et al. (2008) Do theory of mind and executive function deficits underlie the adverse outcomes associated with profound early deprivation?: findings from the English and Romanian adoptees study. Journal of Abnormal Child Psychology 36: 1057-1068.

Conus P, Cotton S, Schimmelmann BG, et al. (2010) Pretreatment and outcome correlates of past sexual and physical trauma in 118 bipolar I disorder patients with a first episode of psychotic mania. Bipolar Disorder 12: 244-252.

Cutajar MC, Mullen PE, Ogloff JR, et al. (2010) Schizophrenia and other psychotic disorders in a cohort of sexually abused children. Archives of General Psychiatry 67: 1114-1119.

Daruy-Filho L, Brietzke E, Lafer B, et al. (2011) Childhood maltreatment and clinical outcomes of bipolar disorder. Acta Psychiatrica Scandinavica 124: 427-434.

Dunne MP, Purdie DM, Cook MD, et al. (2003) Is child sexual abuse declining? Evidence from a population-based survey of men and women in Australia. Child Abuse \& Neglect 27: 141-152.
Figueira ML and Brissos S. (2011) Measuring psychosocial outcomes in schizophrenia patients. Current Opinion in Psychiatry 24: 91-99.

Fisher HL, Craig TK, Fearon P, et al. (2011) Reliability and comparability of psychosis patients' retrospective reports of childhood abuse. Schizophrenia Bulletin 37: 546-553.

Fraser S, Hides L, Philips L, et al. (2012) Differentiating first episode substance induced and primary psychotic disorders with concurrent substance use in young people. Schizophrenia Research 136: 110-115.

Gallagher BJ, 3rd and Jones BJ. (2013) Childhood stressors and symptoms of schizophrenia. Clinical Schizophrenia \& Related Psychoses 7: 124-130.

Glaser D. (2014) The effects of child maltreatment on the developing brain. Medico-Legal Journal 82: 97-111.

Hollingsworth K, Callaway L, Duhig M, et al. (2012) The association between maltreatment in childhood and pre-pregnancy obesity in women attending an antenatal clinic in Australia. PLoS One 7: e51868.

IBM Corporation. (2013) IBM SPSS Statistics for Windows, Version 20.0. Armonk, NY: IBM Corp.

Johns LC, Kompus K, Connell M, et al. (2014) Auditory verbal hallucinations in persons with and without a need for care. Schizophrenia Bulletin 40 Suppl 4: S255-264.

Krug EG, Mercy JA, Dahlberg LL, et al. (2002) The world report on violence and health. Lancet 360: 1083-1088.

Lovibond SH and Lovibond PF. (1995) Manual for the Depression Anxiety Stress Scales. Sydney: Psychology Foundation.

Lysaker PH and Salyers MP. (2007) Anxiety symptoms in schizophrenia spectrum disorders: associations with social function, positive and negative symptoms, hope and trauma history. Acta Psychiatrica Scandinavica 116: 290-298.

Matheson SL, Shepherd AM, Pinchbeck RM, et al. (2013) Childhood adversity in schizophrenia: a systematic meta-analysis. Psychological Medicine 43: 225-238.

Mills R, Scott J, Alati R, et al. (2013) Child maltreatment and adolescent mental health problems in a large birth cohort. Child Abuse \& Neglect 37: 292-302.

Morgan C and Fisher H. (2007) Environment and schizophrenia: environmental factors in schizophrenia: childhood trauma-a critical review. Schizophrenia Bulletin 33: 3-10.

Morrison AP, Frame L and Larkin W. (2003) Relationships between trauma and psychosis: a review and integration. British Journal of Clinical Psychology 42: 331-353.

Nafisi N and Stanley B. (2007) Developing and maintaining the therapeutic alliance with self-injuring patients. Journal of Clinical Psychology 63: 1069-1079.

Norman RE, Byambaa M, De R, et al. (2012) The long-term health consequences of child physical abuse, emotional abuse, and neglect: a systematic review and meta-analysis. PLoS Medicine 9: e1001349.

Obermeier M, Schennach-Wolff R, Meyer S, et al. (2011) Is the PANSS used correctly? a systematic review. BMC Psychiatry 11: 113.

Panzer A. (2008) The neuroendocrinological sequelae of stress during brain development: the impact of child abuse and neglect. African Journal of Psychiatry (Johannesbg) 11: 29-34.

Patterson S, Duhig M, Connell M, et al. (2014) Successful recruitment to a study of first-episode psychosis by clinicians: a qualitative account of outcomes and influences on process. Journal of Mental Health: 1-6.

Ramsay CE, Flanagan P, Gantt S, et al. (2011) Clinical correlates of maltreatment and traumatic experiences in childhood and adolescence among predominantly African American, socially disadvantaged, hospitalized, first-episode psychosis patients. Psychiatry Research 188: 343-349.

Read J, Hammersley P and Rudegai T. (2007) Why, when and how to ask about childhood abuse. Advances in Psychiatric Treatment 13: 9.

Read J, McGregor K, Coggan C, et al. (2006) Mental health services and sexual abuse: the need for staff training. Journal of Trauma \& Dissociation 7: 33-50.

Read J, van Os J, Morrison AP, et al. (2005) Childhood trauma, psychosis and schizophrenia: a literature review with theoretical and clinical implications. Acta Psychiatrica Scandinavica 112: 330-350. 
Schafer I and Fisher HL. (2011) Childhood trauma and psychosis - what is the evidence? Dialogues in Clinical Neuroscience 13: 360-365.

Schenkel LS, Spaulding WD, DiLillo D, et al. (2005) Histories of childhood maltreatment in schizophrenia: relationships with premorbid functioning, symptomatology, and cognitive deficits. Schizophrenia Research 76: 273-286.

Scher CD, Stein MB, Asmundson GJ, et al. (2001) The childhood trauma questionnaire in a community sample: psychometric properties and normative data. Journal of Traumatic Stress 14: 843-857.

Scott J, Chant D, Andrews G, et al. (2007) Association between trauma exposure and delusional experiences in a large community-based sample. British Journal of Psychiatry 190: 339-343.

Sevy S, Robinson DG, Holloway S, et al. (2001) Correlates of substance misuse in patients with first-episode schizophrenia and schizoaffective disorder. Acta Psychiatrica Scandinavica 104: 367-374.

Shevlin M, Dorahy MJ and Adamson G. (2007) Trauma and psychosis: an analysis of the National Comorbidity Survey. American Journal of Psychiatry 164: 166-169.

Spataro J, Mullen PE, Burgess PM, et al. (2004) Impact of child sexual abuse on mental health: prospective study in males and females. British Journal of Psychiatry 184: 416-421.

Stain HJ, Bronnick K, Hegelstad WT, et al. (2014) Impact of interpersonal trauma on the social functioning of adults with first-episode psychosis. Schizophr Bull 40: 1491-1498.

Stain HJ, Galletly CA, Clark S, et al. (2012) Understanding the social costs of psychosis: the experience of adults affected by psychosis identified within the second Australian National Survey of Psychosis. Australian and New Zealand Journal of Psychiatry 46: 879-889.
Stewart G, Sara G, Harris M, et al. (2010) A brief measure of vocational activity and community participation: development and reliability of the Activity and Participation Questionnaire. Australian and New Zealand Journal of Psychiatry 44: 258-266.

Stoltenborgh M, van Ijzendoorn MH, Euser EM, et al. (2011) A global perspective on child sexual abuse: meta-analysis of prevalence around the world. Child Maltreatment 16: 79-101.

Thomas N, Hayward M, Peters E, et al. (2014) Psychological therapies for auditory hallucinations (voices): current status and key directions for future research. Schizophrenia Bulletin 40 Suppl 4: S202-212.

Ucok A and Bikmaz S. (2007) The effects of childhood trauma in patients with first-episode schizophrenia. Acta Psychiatrica Scandinavica 116: $371-377$

Upthegrove R, Birchwood M, Ross K, et al. (2010) The evolution of depression and suicidality in first episode psychosis. Acta Psychiatrica Scandinavica 122: 211-218.

van Dam DS, van Nierop M, Viechtbauer W, et al. (2014) Childhood abuse and neglect in relation to the presence and persistence of psychotic and depressive symptomatology. Psychological Medicine [Epub ahead of print].

Varese F, Smeets F, Drukker M, et al. (2012) Childhood adversities increase the risk of psychosis: a meta-analysis of patient-control, prospectiveand cross-sectional cohort studies. Schizophrenia Bulletin 38: 661-671.

Wang Z, Xue Z, Pu W, et al. (2013) Comparison of first-episode and chronic patients diagnosed with schizophrenia: symptoms and childhood trauma. Early Intervention in Psychiatry 7: 23-30.

World Health Organization. (1992) ICD-10 Classification of Mental and Behavioural Disordrs: Clinical Descriptions and Diagnostic Guidelines. Geneva: World Health Organization. 